\title{
ENDOCRINOLOGÍA PEDIÁTRICA
}

Póster

Determinación de los factores de riesgo en niños y jóvenes de la institución educativa Gimnasio Campestre Marie Curie para el desarrollo de dislipidemias, Bogotá D. C., 2018

\author{
Moscoso JM, Madrigal LJ.
}

Universidad Colegio Mayor de Cundinamarca. Grupo de investigación de Enfermedades Crónicas, Zoonóticas y Adquiridas (ECZA). Bogotá D. C., Colombia.

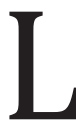

as dislipidemias hacen parte de los factores de riesgo para sufrir enfermedad cardiovascular y constituyen una patología de gran importancia ya que es una de las principales causas de morbimortalidad en personas mayores de 45 años. En la actualidad se observa que una alta cifra de jóvenes presenta signos propios de dislipidemias debido a los malos hábitos alimenticios y a los estilos de vida establecidos desde la niñez. 
El objetivo de la presente investigación es la determinación de factores de riesgo en niños y jóvenes de la Institución Educativa Gimnasio Campestre Marie Curie para el desarrollo de dislipidemias en la ciudad de Bogotá D. C., 2018.

El estudio contó con una muestra poblacional de 90 escolares en edades comprendidas entre los 6 y 16 años, a quienes se les cuantificaron valores de perfil lipídico (lipoproteína de alta densidad [HDL], lipoproteína de baja densidad [LDL] y triglicéridos) además de las medidas antropométricas, después del diligenciamiento de la documentación legal pertinente (consentimiento y asentimiento) y la encuesta de hábitos alimenticios.

Los sueros obtenidos se conservaron a $-20^{\circ} \mathrm{C}$ hasta su procesamiento, se utilizó el kit Spinreact.

Teniendo en cuenta la correlación de valores de referencia publicados por la American Heart Association (AHA) y los datos obtenidos, se concluyó que en la muestra poblacional el 91 \% mostró índices normales de colesterol total, el 67 \% estuvo entre los valores normales de triglicéridos y el 35,3 \% tuvo el colesterol HDL por debajo del valor normal. La tabulación de las encuestas de hábitos mostró que el sedentarismo y los malos hábitos alimenticios aumentan la posibilidad de adquirir dislipidemias.

\section{Póster}

\section{Espectro clínico de una serie de prolactinomas en pediatría}

\author{
Suárez DV, Guzmán EC, Jaramillo JA, Lopera MV, \\ Toro $M$.
}

Departamento de Pediatría y Puericultura, posgrado de Endocrinología Pediátrica, Facultad de Medicina, Universidad de Antioquia. Medellín, Colombia.

$\mathrm{L}$ os prolactinomas son tumores hipofisarios benignos secretores de prolactina; tienen una incidencia $<0,1$ / 1000 000, aunque son infrecuentes en niños; representan el $50 \%$ de los adenomas hipofisarios y el $2 \%$ de los tumores intracraneales. Sus manifestaciones son variadas, pueden ser por efecto de masa, con cefalea y deterioro en los campos visuales; así como por hiperprolactinemia, expresada como galactorrea y pubertad diferida.

Objetivo: caracterizar a un grupo de pacientes con prolactinomas de la consulta externa de endocrinología pediátrica entre 2014 y 2019.

Metodología: descriptiva, retrospectiva.

Descripción: se reportan 6 pacientes, 50 \% mujeres, con un promedio de edad al diagnóstico de 14 años. Todos manifestaron cefalea, $50 \%$ deterioro del campo visual, $33 \%$ galactorrea y solo 1 pubertad diferida. La mayoría tuvo macropro- lactinomas, con niveles de prolactina incluso $>2000 \mathrm{ng} / \mathrm{mL}$; solo un paciente presentó microadenoma, cuyo nivel de prolactina fue el más bajo de la serie ( $56 \mathrm{ng} / \mathrm{mL}$ ). No se demostró la producción de otras hormonas hipofisarias ni otros tumores que hicieran sospechar neoplasia endocrina múltiple (MEN). Todos los pacientes recibieron manejo con cabergolina, con tolerancia y respuesta adecuadas; se les realizó un seguimiento en promedio de 17 meses y se demostró una disminución del tamaño tumoral. Ninguno necesitó manejo quirúrgico.

Conclusión: los prolactinomas son los tumores hipofisarios más frecuentes. En nuestra serie el comportamiento clínico fue variado (cefalea, defectos visuales, retraso de la pubertad, amenorrea y galactorrea). Estos tumores pueden generar efecto de masa e incluso panhipopituitarismo. El nivel basal de prolactina está relacionado con el tamaño tumoral. Los agonistas dopaminérgicos son el tratamiento de elección, debido a que han demostrado buena efectividad y tolerancia, con una reducción del tamaño del tumor y restauración del eje gonadotrópico. Es importante para excluir neoplasia endocrina múltiple tipo 1 (MEN1) y adenoma hipofisario familiar aislado (FIPA).

\section{Póster}

\section{Estado nutricional y riesgo en el metabolismo óseo de niñas escolares en Pasto}

\author{
Ramírez $D^{1}$, Zafrilla $P^{2}$, Granja $M A^{1}$, \\ Arévalo $A C^{1}$, Cerdá $B^{2}$.
}

${ }^{1}$ Hospital Infantil Los Ángeles (HILA). Pasto, Colombia. ${ }^{2}$ Universidad Católica San Antonio de Murcia (UCAM). Murcia, España.

Objetivo: determinar los niveles séricos de vitamina $\mathrm{D}$ en niñas prepuberales sanas de una ciudad del sur de Colombia.

Población de estudio: se incluyeron 50 niñas sanas, con tensión arterial en el p50 \pm una desviación estándar y determinación del estadio puberal de Tanner 1 . Antes de la realización del estudio se obtuvo la aprobación del comité de ética de la investigación. Se incorporaron al estudio luego de la aceptación del menor y la obtención del consentimiento informado del responsable legal.

Resultados: respecto al hemograma, todas las niñas se encontraron dentro de la normalidad, del mismo modo que la albúmina, con una media de 4,85 mg/dL en el grupo estudiado.

Los parámetros bioquímicos de metabolismo óseo, como el fósforo, se encontraron normales con una media de 7,6 g/ $\mathrm{dL}$, del mismo modo que el calcio y el magnesio, con un media de 10,6 mg/dL y 2,03 g/dL, respectivamente. 
Para los niveles de vitamina D, los niveles de 1,25-dihidroxicolecalciferol estuvieron normales con una media de 89,5; no obstante, los niveles de 25-hidroxivitamina D se encuentran en una media de 22,5 ng/mL con una mínima de 13,9 y una máxima de 37,6 con una desviación estándar de 5,3.

Conclusiones: en este trabajo se encontraron todos los metabolitos séricos analizados dentro de la normalidad para el grupo estudiado, a excepción de la vitamina D, que en los niveles de calcidiol (25-hidroxivitamina D) se encontró deficiencia de esta vitamina, esto puede asociarse con falta de ingesta en la dieta, exposición a la luz solar o poca actividad física.

\section{Póster}

\section{Síndrome metabólico y sus componentes individuales, según el sexo y criterios de definición en una cohorte de niños y adolescentes de Bucaramanga}

\section{Serrano $N^{1}$, Villa-Roel $C^{2}$, Gamboa-Delgado EM', Quintero-Lesmes DC}

${ }^{1}$ Fundación Cardiovascular de Colombia (FCV). Bucaramanga, Colombia. ${ }^{2}$ Faculty of Medicine \& Dentistry-Emergency Medicine Division, University of Alberta.

${ }^{3}$ Universidad Industrial de Santander (UIS).

Objetivo: evaluar las prevalencias de síndrome metabólico (SM) y sus componentes individuales, según sexo y criterios de definición entre la línea basal y el seguimiento.

Diseño del estudio: estudio tipo panel, anidado en una cohorte poblacional.

Lugar del estudio: Bucaramanga, Colombia.

Pacientes/sujetos: niños(as) y adolescentes (n línea basal = 1282; n seguimiento $=494$ ).

Mediciones: variable dependiente: SM definido por los criterios National Cholesterol Education Program Adult Treatment Panel III (ATP III) e International Diabetes Federation (IDF). Variables independientes: sexo, edad, nivel socioeconómico, duración de la lactancia materna; peso, talla, índice de masa corporal (IMC), circunferencia de cintura, circunferencia de la cadera, índice cintura-talla, índice cinturacadera, actividad física moderada e intensa, tiempo frente a las pantallas; colesterol total, lipoproteína de baja densidad (LDL), insulina en ayunas y el índice modelo homeostático para evaluar la resistencia a la insulina (HOMA-IR). Se usaron las pruebas estadísticas T de Student o U de Mann Whitney, de acuerdo con la distribución de las variables. Todos los datos se analizaron en el programa estadístico Stata, versión 14.0.
Resultados: se presentaron diferencias estadísticamente significativas por sexo en el seguimiento en cuanto a la obesidad abdominal $(\mathrm{p}=0,002)$ y glucemia en ayunas alterada ( $\mathrm{p}$ $=0,046)$, de acuerdo con el criterio ATP III; y con respecto a la obesidad abdominal ( $\mathrm{p}=0,002)$, hipertensión arterial ( $\mathrm{p}$ $=0,015)$, triglicéridos $(\mathrm{p}=0,039)$ y glucemia en ayunas $(\mathrm{p}=$ 0,046) según el IDF.

Conclusiones: la prevalencia de SM fue de 9,89\% en niñas y 9,16 \% en niños, en la línea basal, la cual se incrementó después de 10 años a 11,42 \% en niñas y 15,00 \% en niños. La prevalencia de obesidad abdominal fue mayor en las mujeres según ambos criterios; mientras que las de hipertensión arterial, hipertrigliceridemia y alteración de glucemia fueron mayores en los hombres.

\section{Póster Valoración de estilos de vida saludable de una población escolar de estrato medio en la ciudad de Bogotá D. C.}

\author{
Cajiao V, Rodríguez S, Castro LP, Prieto C, Munar L, \\ Aivasovsky I, et al.
}

Gimnasio Yacard, Universidad de La Sabana. Bogotá D. C., Colombia.

$\mathrm{E}$ n Colombia se ha prestado gran atención a la situación nutricional infantil debido a las consecuencias que pueden desarrollarse, dicha situación puede propiciar enfermedades crónicas no transmisibles como obesidad, diabetes, hipertensión, enfermedades coronarias, entre otras. El objetivo de este trabajo es determinar el estado nutricional de una muestra en una población escolar en Bogotá D. C. de estrato medio, teniendo en cuenta los hábitos alimenticios, actividad física, niveles de micronutrientes y bioquímica sanguínea.

La metodología consistió en la realización de un estudio de corte transversal en el que se analizaron los hábitos nutricionales y estilos de vida de un grupo de 25 estudiantes entre los 6 y 12 años, y se correlacionaron dichos datos con los parámetros bioquímicos tomados.

Según los resultados obtenidos, se evidenció deficiencia de micronutrientes como vitaminas, minerales y oligoelementos en edades preescolares y escolares. Respecto a sus hábitos nutricionales se demostró un bajo consumo de vitaminas A, B6 y B9, bajo consumo de oligoelementos, frutas y verduras, un elevado índice de sedentarismo y altos niveles de grasa corporal. 
Se puede concluir que, a pesar de ser una población con los recursos económicos necesarios para financiar una alimentación saludable, existe malnutrición por la carencia de nutrientes o exceso de algunos.

Es importante hacer visible esta problemática con el fin de ejecutar las acciones necesarias para prevenir, atender y con- trolar el efecto de la malnutrición por medio de la sensibilización de padres y representantes y, de este modo, mejorar los estilos de vida y hábitos saludables de los estudiantes escolares y sus familias como método preventivo para el desarrollo de enfermedades crónicas. 